\title{
La perspective des coûts de transaction perçus : une explication de l'intermédiation immobilière
}

Papier accepté pour la revue dấconomie régionale et urbaine 3, 2014

\section{Fabrice LARCENEUX}

Chercheur CNRS,

DRM, Université Paris Dauphine

fabrice.larceneux@dauphine.fr

\section{Thomas LEFEBVRE}

Doctorant

DRM, Université Paris Dauphine

tlefebvr@gmail.com

\section{Arnaud Simon}

Maitre de conférences,

DRM, Université Paris Dauphine

arnaud.simon@dauphine.fr

Mots clef : coûts de transaction, intermédiation, marché immobilier résidentiel

Keywords: intermediation, real estate housing, transaction cost 


\section{Résumé}

L'objectif de cette recherche est d'étudier les déterminants du recours aux solutions directes ou intermédiées sur le marché immobilier résidentiel français. La perspective théorique des coûts de transaction perçus fournit un cadre utile pour appréhender lâxistence dồn mode de transaction dans le secteur immobilier. A partir de l'analyse de 4142 questionnaires portant sur des transactions effectivement réalisées, les modèles Logit mis en î uvre montrent que l'intermédiation immobilière ne s'explique pas par les caractéristiques physiques ou spatiales du bien, ni par des variables socio-démographiques des acteurs, mais davantage par les perceptions qu'ont les acheteurs et vendeurs de l'intérêt des agents immobiliers et des bénéfices qu'ils peuvent en retirer. Six bénéfices perçus se révèlent pertinents pour mieux comprendre l'existence des agents immobiliers dans un contexte de nouvelles technologies où les relations directes sont aujourd'hui largement facilitées.

\section{Summary}

The aim of this research is to study the determinants of the use of direct versus intermediated solutions on the French housing market. The theoretical perspective of perceived transaction costs provides a useful framework for understanding the existence of a specific mode of transaction in real estate. From the analysis of 4142 questionnaires on transactions actually carried out, the logit models used show that the real estate intermediation can not be explained by physical or spatial characteristics of the property, or by socio-demographic variables, but more by perceptions of buyers and sellers in the interest of real estate agents and the benefits they can derive. Six perceived benefits prove relevant for understanding the existence of Realtors in the context of new technologies where direct relationships are now facilitated. 


\section{- 1 - Introduction}

Dans l'immobilier, comme dans d'autres secteurs, la généralisation de l'utilisation d'internet pose aujourd'hui la question cruciale de l'existence des intermédiaires. La présence ou non d'intermédiaires renvoie à deux logiques distinctes de marché : Dans les marchés centralisés, un ou plusieurs acteurs économiques î uvrent comme des intermédiaires de marchés entre vendeurs et acheteurs. Il s'agit par exemple de réseaux de distribution, de courtiers, de maisons dênchères, etc. En immobilier, ces intermédiaires fournissent de l'information aux consommateurs, information dont une part importante concerne l'évaluation qui est faite du bien (HÄNCHEN, UNGERN-STERNBERG, 1985 ; SASS, 1984), et organisent la transaction (BAILEY, 1996 ; HACKET, 1992). Dans un monde complexe, ces agents immobiliers sont supposés sélectionner les interactions optimales et améliorer la coordination entre les vendeurs et acheteurs (BALISH, RICHARTZ, 1967), contribuant à créer ce que les économistes nomment l'équilibre de marché. A l'inverse, dans les marchés décentralisés, acheteurs et vendeurs se rencontrent directement sur des espaces dédiés. A l'image des petits marchés locaux ou des bazars du moyen orient, il peut exister un grand nombre d'interactions entre les parties et aucun intermédiaire pour les organiser. Et c'est cette structure de marché qui est aujourd'hui largement facilitée par internet, et qui constitueraient une menace pour les agents immobiliers. En France, les consommateurs, i.e. les acheteurs et les vendeurs de biens immobiliers, peuvent en effet faire le choix d'utiliser ou non les services d'intermédiation.

"Plus d'une transaction sur deux se déroule directement entre particuliers" proclame une publicité française pour un site internet entre particuliers. Avec la diffusion généralisée de l'information sur internet, il est en effet, en théorie, devenu aujourd'hui plus facile pour les acheteurs de rechercher de l'information, des biens à vendre et d'opérer des transactions directement avec les vendeurs sur ce type de plateformes. Si les consommateurs ont libre choix d'utiliser les services d'un agent immobilier pour effectuer une transaction, cette intermédiation est supposée fortement décroitre à mesure que les acteurs s'approprient directement l'information (TuCILlo, 1997). Dans la lignée des travaux de WiLliamson (1975), la contractualisation avec des intermédiaires, synonyme d'un mauvais fonctionnement du marché, serait amenée à disparaitre à mesure que le marché tend à devenir plus efficace. Cette perspective inviterait donc à considérer d'un point de vue général l'existence des intermédiaires comme temporaire : S'ils sont encore présents, c'est qu'ils bénéficient quelques temps de leur rente d'information, remplissant des tâches inutiles et financièrement coûteuses pour les consommateurs. Mais est-ce réellement le cas ? Une dizaine d'année après la généralisation d'Internet auprès du grand public, un constat s'impose : acheteurs et vendeurs ne constituent pas un marché unique, direct et décentralisé alors qu'internet est supposé leur permettre cette rencontre mutuellement profitable.

Se pose alors la question des raisons de l'existence actuelle des agents immobiliers : ces intermédiaires apporteraient des services différenciant des plateformes de relations directes et attendus par les consommateurs, acheteurs et vendeurs. Ces services constitueraient une réelle plus-value économique, d'autant plus utile que le marché est peu fluide et ne permet pas facilement aux offreurs et demandeurs de se rencontrer, i.e. lorsque la densité des acheteurs et des offres n'est pas suffisante.

Si la littérature a relativement bien exploré l'influence des agents sur les prix de vente (BAJTELSMIT, WORZOLA, 1997 ; ViOLAND et al., 2007) ou sur le temps de recherche d'un bien 
ou d'un acheteur (ELDER et al., 2000), peu de recherches se sont positionnées du point de vue du consommateur pour comprendre les déterminants du choix d'une transaction intermédiée plutôt que non intermédiée. Faisant écho à l'appel lancé par CROZIER et MC LEAN (1997), cette recherche vise à combler ce manque et propose des éléments d'explication via la perspective des coûts de transactions perçus : le recours aux agents immobiliers s'expliquerait non pas par des caractéristiques physico-spatiales des biens ou sociodémographiques des individus, mais plutôt parce qu'acheteurs et vendeurs considèrent que les agents immobiliers proposent des services utiles à leurs yeux, i.e. qui diminuent des coûts de transaction qu'ils perçoivent comme importants. Dès lors, cette analyse se positionne délibérément au niveau du preneur de décision et non au niveau sectoriel, afin d'étudier ses attentes en matière de services d'intermédiation. Dans un premier temps, un cadre conceptuel fondé sur les bénéfices attendus est proposé. Dans un second temps, le modèle, testé empiriquement, montre que six raisons majeures peuvent aujourd'hui expliquer la persistance de l'intermédiation immobilière. Ce papier se termine par une discussion des résultats et propose des voies de recherches.

\section{- 2 - \\ Revue de littérature et cadre conceptuel}

\subsection{De la rente d'information aux coûts de transaction perçus par les consommateurs de services}

Contrairement aux achats quotidiens en grande consommation, acheter ou vendre un logement constitue une transaction financièrement, matériellement et symboliquement risquée (GIBLER, NELSON, 2003 ; LARCENEUX, PARENT, 2010 ; NASAR, 1989). Il s'agit souvent du plus important engagement qu'un individu réalise dans sa vie (en France, de l'ordre de $23 \%$ du budget des familles ï ACCARDO, BugETA (2009) - et 25\% selon l'Institut national français de la statistique) dans un lieu porteur de sens. En outre, cette transaction implique d'avoir un certain degré de connaissances juridiques et d'informations, notamment sur les prix de marché. Enfin, en plus des durées légales incompressibles, le temps dévolu à la recherche du bien ou de l'acheteur solvable, à la négociation et à la signature du compromis de vente sont généralement évalués à environ plus de trois mois (entre 12 et 15 semaines selon BARYLA et al., 2000). La transaction immobilière est donc très spécifique car beaucoup plus risquée, complexe et longue que les autres types de transactions. Les logiques d'analyse qui s'appliquent à d'autres secteurs ne seraient donc pas forcément pertinentes dans le secteur immobilier.

Dans l'optique d'une transaction immobilière, la contractualisation d'un individu avec un intermédiaire peut s'inscrire dans les réflexions de WiLliamSON $(1975,1981)$ qui justifie l'existence d'une coordination d'acteurs en dehors du marché du fait de défaillances de marché. Le processus marchand, compliqué et coûteux, comporte de fortes incertitudes entre individus, tentés quelques fois de garder l'information, voire de la déformer à leur profit (COASE, 1937).

Dans la mesure où conclure une transaction immobilière implique de connaître des informations relativement difficiles à obtenir, nécessite du temps et des compétences, le coût élevé de ce travail justifiait jusqu'à présent l'existence d'intermédiaires (ZUMPANO et al., 2002). Cette existence fondée sur une asymétrie d'information s'est enracinée sous la forme "d'arrangements institutionnels" spécifiques au sens de WiLliaMSON (1981). Accompagnant cette complexification de la législation et celle du marché immobilier, les intermédiaires se sont structurés autour de réseaux d'agences ou de franchises organisant le lien entre acheteurs et 
vendeurs. Aujourd'hui, un des moyens de pérenniser cette position d'intermédiaire est de s'assurer, de manière incitative ou coercitive, l'accès aux biens via la captation des mandats des vendeurs. Incitative car les frais d'agence, potentiellement imputables aux deux parties sont en réalité en totalité à la charge de l'acheteur ; coercitive, via les mandats exclusifs qui protègent l'intermédiaire des comportements opportunistes d'un vendeur qui utiliserait les services (non payables à l'avance) pour vendre in fine en direct. Cette situation fait dire de manière un peu provocatrice à LEVITT et DUBNER (2005) que ces réseaux doivent leur existence du fait de la "rente d'information" qu'ils se sont accaparés et qu'ils cherchent à préserver, depuis la connaissance de l'existence du bien jusqu'à celle du prix de marché et des connaissances techniques et légales. Les intermédiaires fonderaient ainsi leur existence sur une captation de l'offre d'une part et sur la rente d'information d'autre part, alors même qu'ils ne disposent pas de toute l'information (BONNEVAL, 2011).

L'utilisation d'internet tend cependant à transformer les modèles d'affaires traditionnels : l'intermédiaire y est perçu comme un simple élément de la chaine de valeur, sans grande valeur réelle, mais qui in fine augmente les coûts et le prix des biens. La prime de prix représentée par les frais d'agence serait aujourd'hui difficile à justifier dans un contexte où l'information circule librement : des technologies nouvelles, internet ou mobiles, permettent aux vendeurs et acheteurs de se rencontrer directement, de manière plus fluide qu'avant. Internet éroderait ainsi le pouvoir de marché des agents immobiliers au profit des particuliers (BAEN, GUTTERY 1997 ; TUCILLIO 1997) : la connaissance des biens à vendre, les prix de marché, les compétences juridiques nécessaires sont aujourd'hui à la portée de n'importe quel acteur quasi-gratuitement. Et, de fait, dans de nombreux secteurs, les comportements de recherche se font directement sur le web. Internet représentent $80 \%$ des recherches immobilières en France en 2013 (FF2I) et les sites de transaction directe "Consumer to Consumer" (C2C) sont en croissance régulière. A titre d'exemple, Internet a permis au classique journal papier de petites annonces de se transformer en sites de référence (pap.fr, le boncoin.fr, etc.) qui offrent un très grand nombre d'annonces en direct. Une partie de ces sites organise la promotion des biens et fournit aux vendeurs et acheteurs de l'information, tels que les prix moyens, les calculs de taux d'intérêt, des éléments juridiques, etc. En 1995, BENJAMIN et WIGAND avaient ainsi prédit une forte diminution des intermédiaires du fait de l'arrivée d'internet et l'augmentation des plateformes internet C2C. Le vendeur devait récupérer logiquement une plus grande partie du profit et l'acheteur bénéficier d'un plus large choix et d'un prix plus faible.

Pourtant, une dizaine d'année après l'arrivée du réseau internet auprès du grand public, il existe toujours des intermédiaires dans l'immobilier : en France, ces acteurs représentent un véritable poids économique (35 000 entreprises, 18 milliards dêuros de CA en 2012) qui se traduit en parts de marché : estimée aux alentours de 84\% aux Etats-Unis (BARYLA et al., 2000), elle est de l'ordre de 70\% en France selon Violand et al. (2007). Ce chiffre est similaire à ce que la présente étude a pu établir (plus de 70\%, sur plus de 4000 transactions analysées, une première en France). La prédiction de l'avènement d'un marché décentralisé se révèle ainsi erronée.

Les intermédiaires continuent donc bel et bien d'exister grâce aux systèmes de pérennisation incitatifs et coercitifs mis en place, mais ce ne sont pas là les seules raisons. En France, contrairement aux Etats-Unis, le marché n'est pas régi par les mandats exclusifs (on en compte moins de $10 \%$ des mandats) et le vendeur pourrait facilement espérer récupérer le profit avec un comportement opportuniste de vente. Comment dès lors expliquer qu'acheteurs et vendeurs aient encore recours aux intermédiaires sur le marché français ? 
La théorie des coûts de transaction fournit une perspective pertinente pour appréhender la problématique d'existence d'un mode de transaction privilégié dans un secteur donné. Si l'intermédiaire peut supplanter le recours direct au marché, c'est en raison des économies de coûts de transaction qu'il permet de réaliser. Cette perspective s'attache à identifier de manière objective, par des économistes spécialistes du secteur, la nature des coûts de transaction dans un secteur donné (WILLIAMSON, 1975) : Ces coûts de transaction sont définis comme "la somme des coûts ex ante de négociation et de rédaction du contrat qui relie les deux entités et des coûts ex post d'exécution et de mise en vigueur et de modification des contrats lorsqu'apparaissent des conflits" (WILLIAMSON, 1981). Les coûts de transaction ex-ante correspondent donc à la première étape de la transaction : prospection, négociation, recherche de partenaires et établissement de lâvant-contrat, i.e. le compromis de vente. Les coûts de transaction ex post concernent les coûts de surveillance et de contrôle après la signature. Dans le secteur immobilier, les coûts dits ex ante sont les plus pertinents pour appréhender les raisons de lâxxistence dôn mode de transaction privilégié. En effet, une juridiction d'Etat - les notaires est chargée de s'assurer de la bonne application du contrat de vente.

Les agents économiques sont amenés à choisir des arrangements contractuels qui leur permettent de minimiser ces coûts de transaction (BROUSSEAU, 1989). Dans la perspective de l'économie institutionnelle, WILLIAMSON (1985) fait des spécifications objectives a priori de la transaction l'explication du mode de gouvernance optimal. Ce mode de gouvernance unique sélectionné dans un secteur dépendrait donc de l'évaluation des coûts préalables liés à la réalisation de la transaction. Ces coûts sont fonction du degré de spécificité des actifs, de la fréquence de la transaction et du degré d'incertitude interne et externe liée à la transaction. L'analyse a posteriori conduit ainsi à définir un mode de contractualisation unique qui devrait tendre soit vers le recours direct (market), soit vers l'intégration et l'internalisation (hierarchy) en fonction des spécifications objectives identifiées du secteur.

Or, le mode de gouvernance d'un secteur résulte aussi des comportements individuels, i.e. des contrats passés entre individus. Ce sont donc potentiellement les "déterminants" des actions des individus qui permettent d'expliquer l'émergence d'un type de transaction plutôt qu'un autre. Dans la perspective de la théorie de l'action raisonnée, les déterminants sont les attitudes que manifestent les individus par rapport à une option particulière et qui sont susceptibles d'expliquer un comportement de choix (FISHBEIN, AJZEN, 1975 ; KROSNICK, 1989). L'attitude envers une option dépend des croyances des individus sur les bénéfices et les coûts qu'ils escomptent retirer du choix de cette option plutôt qu'une autre. Mais en réalité, l'individu n'a pas de connaissances précises ex ante de la satisfaction du service proposé. Doté d'une rationalité limitée (SIMON, 1991) et d'une connaissance imparfaite des procédures et des conditions de la transaction, il imagine et perçoit les avantages et inconvénients de chaque solution, i.e. les coûts et les bénéfices qu'il peut escompter de chaque alternative. Il ne s'agit donc pas, dans ce présent article, d'une analyse extrinsèque objectivée a posteriori des coûts de transaction mais d'une analyse des perceptions subjectives qu'en a lôndividu, perceptions qui expliquent le choix d'un mode de gouvernance spécifique, i.e. le recours à un agent immobilier ou la relation directe. Affirmant ce positionnement, cette recherche se propose de prolonger la théorie des coûts de transaction de WILLAMSON au niveau individuel, en considérant les coûts de transaction perçus par les acteurs. 
Dans cette perspective théorique des coûts de transaction perçus, l'alternative make vs. buy (RANGAN et al., 1992) renvoie à la dichotomie market vs. hierarchy de WILLIAMSON : soit lôndividu décide de s'adresser directement au marché et supporte lui-même tous les coûts non monétaires (market), soit il décide d'acheter les services d'intermédiation et intègre les coûts de transaction sous la forme d'un contrat avec un agent (hierarchy). Le coût de transaction est alors objectivé d'un point de vue monétaire : les services sont généralement facturés de $4 \%$ à $9 \%$ selon le montant de la vente et représentent le coût d'opportunité de ne pas avoir recours au marché. Dans leur calcul économique, acheteurs et vendeurs devraient refuser les services d'un agent immobilier dès lors que le sacrifice financier est perçu comme trop important au regard des bénéfices apportés. Comment modéliser cette perception ? Quels sont vraiment ces bénéfices pris en compte par les décideurs ? La littérature en économie immobilière n'est pas très prolixe sur ce sujet. Elle s'est essentiellement organisée autour de l'impact des agents immobiliers sur les types de transactions immobilières. Et les résultats obtenus ne permettent pas de conclure à un effet particulier : les caractéristiques des agents n'ont pas d'effets sur les prix de vente des logements, ni sur les temps moyens passés par les biens sur le marché (JUD, WinKLER, 1994 ; JUD et al., 1996 ; MUNNEKE, YAVAS, 2001 ; YANG, YAVAS, 1995 ; ZUMPANO et al., 1996). Plus spécifiquement, certaines recherches explorent les raisons du choix d'un agent immobilier plutôt qu'un autre : la capacité de l'agent à être en phase le vendeur, la réputation de l'agence, la connaissance et les compétences de l'agent seraient ainsi les facteurs les plus importants (JOHNSON et al., 1988). Dâuutres recherches abordent ces problématiques sous lângle de la sociologie économique en sônterrogeant sur le rôle des intermédiaires dans lôpppariement de lôffre et la demande de biens immobiliers (BONNEVAL, 2011) : lômportance du relationnel dans le métier dôgent immobilier contribue à construire la notoriété de lôntermédiaire dont la bonne image est déterminante dans lôbtention et la conduite des transactions marchandes. Mais aucune recherche ne formalise réellement les raisons qui poussent un individu à choisir de payer pour les services d'un agent immobilier plutôt que de passer par des relations directes.

Cette théorie des coûts de transaction perçus propose que les individus prennent leur décision make vs. buy en comparant ce qu'ils pensent des coûts de transaction a priori pour eux (WALKER, WEBER, 1984). Répondant à ces coûts de transaction, les agents immobiliers proposent des services qui ont pour particularité d'être intangibles, inséparables, hétérogènes et périssables, donc très difficilement quantifiables avant qu'ils ne soient rendus. Il s'agit donc ici de centrer l'analyse sur les perceptions qu'ont les consommateurs de la capacité des intermédiaires à répondre aux coûts non monétaires liés à la conclusion d'une transaction. Lorsque ils sont évalués comme supérieurs aux coûts monétaires demandés par l'intermédiaire, le décideur fait le choix du buy, i.e. d'un mode de transaction "internalisé" et il a recours à un agent immobilier. A l'inverse, s'il considère que l'aspect financier de la transaction est significativement moins avantageux lorsqu'il y a un agent immobilier font le choix du make et doivent avoir tendance à vendre plutôt en direct.

\subsection{Bénéfices perçus des intermédiaires et choix de transaction : un cadre conceptuel}

Afin de faire émerger les bénéfices de chaque mode de gouvernance, quinze entretiens qualitatifs ont été menés avec des consommateurs potentiels et cinq entretiens avec des intermédiaires professionnels de l'immobilier résidentiel. Une première partie du guide d'entretien évaluait les difficultés perçues pour aboutir à la signature d'un contrat de vente et 
une deuxième partie interrogeait les apports éventuels des intermédiaires. L'analyse des entretiens montre que les coûts psychologiques perçus lors d'une transaction immobilière se structurent autour de deux grandes dimensions pour lesquelles les individus anticipent sur chacun des trois types de risques :

- d'une part, la dimension processus de recherche d'information qui intègre le risque de ne pas trouver le bien ou l'acheteur conforme aux attentes, le risque de ne pas trouver rapidement et le risque d'être fortement contraint par l'organisation de la transaction (appels, visites, etc. ) ;

- d'autre part, la dimension gestion de la transaction qui intègre le risque que la transaction puisse ne pas bien se dérouler, le risque de ne pas savoir mener à bien tout seul la négociation et de ne pas avoir les éléments nécessaires pour convaincre (négociation du prix, rédaction de lôvant-contrat etc.) et le risque que la solution finalement retenue ne soit pas financièrement intéressante (surévaluation des biens en solution directe ou importance perçue de la commission).

Face à ces six niveaux de risques perçus, les professionnels de l'immobilier évoquent souvent la "compétence" ou "l'expertise" de l'agent immobilier. Toutefois cet élément n'est pas, en soi, un bénéfice pour le consommateur mais un moyen d'obtenir ce qu'il recherche. L'analyse des entretiens a permis d'isoler les bénéfices utiles, effectivement attendus. Pour chacun d'entre eux, il est ainsi possible de faire l'hypothèse qu'ils influencent de manière significative le choix d'avoir ou non recours à un agent immobilier.

- Le bon matching : Concilier le budget et les désirs se révèle la tâche la plus difficile lors d'une recherche de logement (sondage PAP, 2012) : 57,2\% de Français ont même considéré cet épisode comme leur pire cauchemar. De plus, son aboutissement n'a pas forcément été satisfaisant, puisque $58 \%$ de personnes interrogées se disent mécontentes de leur résidence actuelle. Durant la phase de rencontre et d⿳⺈tablissement des termes du contrat, lâgent immobilier mandaté par le vendeur fournit à lộcheteur le bien recherché potentiellement en adéquation avec ses attentes; et au vendeur, un acheteur solvable. (BARYLA, ZUMPANO, 1995). Trouver une solution conforme aux attentes est un des éléments attendus comme devant être plus performant que ne le permettrait un site de transactions directes (JUD, FREW, 1986).

- Le gain de temps : Variable souvent explorée dans la littérature immobilière, le temps dédié à l'achat (ou le temps de présence du bien sur le marché - time on market) est une donnée objective qui serait fortement réduite par un agent immobilier (RUBINSTEIN, WOLINSKY, 1987 ; YAVAS, 1994). En effet, l'agent immobilier augmente le nombre de logements potentiellement adaptés pour un acheteur (BARYLA, ZUMPANO, 1995 ; ELDER et al., 1998 ; ZUMPANO et al., 2000) et a intérêt à vendre vite le bien qui lui a été confié. Le bénéfice perçu est, tant pour l'acheteur que pour le vendeur, le fait qu'ils pourront signer la transaction plus rapidement via un agent immobilier que via une solution directe.

- L'absence de contraintes : Lorsqu'ils considèrent que le processus est contraignant, les individus ont tendance à chercher des solutions qui leur simplifient la tâche, qui leur demandent moins d'efforts (EDLAND, SVENSON, 1993). Le bénéfice perçu est le fait que l'agent immobilier permet de minimiser les contraintes imposées par la transaction en prenant à sa charge 
l'ensemble de l'organisation (e.g. présélection des biens pertinents, organisation des visites et vérification de la solvabilité des acheteurs).

- La réassurance : MCKENZIE et BETTS (1994) ont montré que les consommateurs avaient recours aux intermédiaires dès lors que la situation était perçue comme trop complexe. A nouveau, l'agent immobilier les rassure par sa capacité à appréhender cette complexité. Le bénéfice perçu serait d'être rassuré sur l'ensemble des risques inhérents à la transaction (HISCOCK et al. 2001) de manière plus importante via des agents immobiliers que via des sites de transactions directes. Cette réassurance perçue recouvre aussi l'anticipation de coûts de transaction ex post tels que définis par WILLIAMSON, à savoir les coûts résultant de la survenance de problèmes et de conflits.

- La négociation facilitée : La vente immobilière est une négociation entre des parties aux intérêts divergents : lâcheteur veut acheter le moins cher possible et le vendeur veut vendre le plus cher possible. La dimension affective du bien pour son occupant est aussi un obstacle à la bonne évaluation du prix. La présence dôn intermédiaire, supposé expert et soucieux des intérêts de chacune des parties, évite que les divergences ne se transforment en conflit. Le bénéfice perçu est le fait que lôggent immobilier joue un rôle de négociateur dans la dernière phase de la transaction et qu'il n'est pas plus facile de négocier en direct avec le vendeur ou l'acheteur qu'avec l'intermédiaire immobilier. L'agent immobilier devrait permettre de faciliter la négociation entre les parties pour arriver à un accord.

- Le caractère coûteux de la commission : La relation intermédiée, ou plus exactement la délégation de transaction, est une situation où les acteurs demandent à des tiers de travailler dans leur intérêt en contre-partie d'une commission monétaire censée valoriser le travail effectué. Il s'agit alors d'une analyse coûts/bénéfices permettant à l'acheteur ou au vendeur d'explorer dans quelle mesure il lui parait plus ou moins pertinent de choisir une solution intermédiée plutôt que directe. En France, les frais d'agences classiques, qui se situent entre 4\% et 9\%, sont souvent décriés comme étant les plus élevés d'Europe. La perception d'un coût élevé par rapport aux services rendus correspond à l'idée que l'agent immobilier ne représente pas une solution financièrement intéressante.

Le recours aux agents immobiliers pourrait s'expliquer aussi par d'autres dimensions que la perception subjective des acheteurs et vendeurs. Il s'agit des variables objectives classiquement étudiés dans les recherches en économie immobilière telles que les caractéristiques du bien (le type de bien, le nombre de pièces, la localisation géographique) ou celles de l'individu (le statut matrimonial, l'âge, le revenu). Une analyse empirique permet d'explorer la pertinence de cette perspective théorique des coûts de transaction perçus comme explicative des comportements de choix des individus.

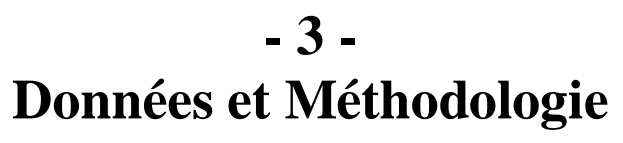


La plupart des recherches empiriques en économie de lômmobilier utilisent généralement lônformation sur le type de transaction (intermédiée ou non) en tant que variable de contrôle de son effet sur le prix des biens. Nous proposons ici dêxplorer les déterminants du recours aux solutions intermédiées sur le marché immobilier résidentiel français. A lônformation décrite par les caractéristiques physiques et spatiales des biens et sociodémographiques des individus, utilisées classiquement dans ces champs de recherche, sont ajoutées des variables sur la perception qu'ont les consommateurs des services immobiliers.

Afin de tester l'ensemble des hypothèses, une collecte de données a été menée directement auprès d'acheteurs et de vendeurs via internet à partir dôun questionnaire établi pour répondre à notre étude. Les premières questions ont été construites de manière à déterminer lâxpérience immobilière du répondant : utilisation de différents canaux de prospection (plateformes de transaction directes et/ou agences immobilières), contractualisation finale de la transaction (plateformes de transaction directes ou agences immobilières), nature de la transaction (achat ou vente). Puis les caractéristiques physiques et spatiales des biens ont été recueillies : le type de bien (appartement, maison), le nombre de pièces (studio/2 pièces, 3 pièces, 4 pièces et plus), la localisation France (Paris, petite couronne, grande couronne ou province) et la centralité (centre-ville, périphérie, campagne). Enfin, des éléments sociodémographiques sur le répondant ont été récoltés tels que l'âge, le statut matrimonial (célibataire, en couple, avec un ou des enfants) et le revenu du foyer. Le reste du questionnaire est consacré aux bénéfices perçus du fait de contractualiser via un agent immobilier par rapport au fait de passer par une transaction entre particuliers). Ces variables ont été mesurées via des échelles de Likert classiques en sept points (de pas du tout d'accord à tout à fait d'accord).

Il a ainsi été demandé aux répondants de donner leur degré d'accord via les questions suivantes : Comparé à la vente directe entre particuliers (pap.fr, leboncoin.fr, etc.), é

$\checkmark$ Côst plus sûr passer par une agence immobilière (Réassurance)

$\checkmark$ On gagne du temps en passant par une agence immobilière (Gain de temps)

$\checkmark$ Côest moins contraignant de passer par une agence immobilière (Minimisation des contraintes)

$\checkmark$ Côst financièrement beaucoup moins intéressant de passer par une agence immobilière (Intéressant financièrement)

$\checkmark$ Un agent immobilier me permet de négocier plus facilement (Négociation)

$\checkmark$ Un agent immobilier me permet de trouver plus facilement un bien me correspondant / un acheteur sérieux (Matching)

Le questionnaire a été envoyé à 180248 individus ayant recherché de l'information immobilière via le site internet Meilleursagents.com qui diffuse des informations sur les prix des logements. 99\% des individus s'inscrivant sur le site sont de simples visiteurs qui recherchent des estimations des biens et qui ne passent aucun de contrat avec le site. 3992 individus $(2,2 \%)$ ont répondu entièrement au questionnaire ${ }^{1}$. Le tableau ci-dessous présente les effectifs obtenus. A notre connaissance, il s'agit de la première enquête de ce genre en Europe. De manière analogue, en 1996, ZUMPANO et ses collègues avaient interrogés 30 000 ménages américains et avaient recueilli 2495 observations, sur les acheteurs uniquement.

$\mathrm{C} 2 \mathrm{C}$

\footnotetext{
${ }^{1}$ Questionnaire complété entièrement sans données aberrantes et/ou erreurs de saisies.
} 


\begin{tabular}{cccc}
\hline Achat & 433 & 1637 & 2070 \\
Vente & 467 & 1455 & 1922 \\
\hline Total & 900 & 3092 & 3992
\end{tabular}

\section{Tableau 1 : Nombre d'individus interrogés selon que la transaction a été signée sur un mode non intermédié (C2C) ou intermédié (AI)}

Le premier résultat de cette enquête est que, durant la phase de prospection, $70 \%$ des personnes interrogées déclarent avoir utilisé à la fois les sites de transactions directes et les agences immobilières. Le parcours de recherche est donc largement extensif et non seulement sur un mode spécifique, reflet entre autres des possibilités pour certains vendeurs de conclure un mandat avec une agence tout en gardant la possibilité de vendre en direct (BONNEVAL, 2012). Second résultat, on note qu'une forte proportion de notre échantillon a réalisé une transaction via un agent $(77 \%)$. Cette proportion se révèle stable sur une très grande partie des caractéristiques des biens et des individus. Le tableau 2 présente les effectifs (et proportions) obtenus pour les deux groupes sur l'ensemble des variables.

C2C AI

\begin{tabular}{lcc}
\hline Caractéristiques des biens & & \\
\hline Appartement & $670(23 \%)$ & $2223(77 \%)$ \\
Maison & $230(21 \%)$ & $869(79 \%)$ \\
\hline Studio/2pièces & $281(23 \%)$ & $946(77 \%)$ \\
3 pièces & $239(23 \%)$ & $816(77 \%)$ \\
Plus de 4 pièces & $380(22 \%)$ & $1330(78 \%)$ \\
\hline Paris & $295(24 \%)$ & $959(76 \%)$ \\
PC & $255(24 \%)$ & $799(76 \%)$ \\
GC & $155(23 \%)$ & $519(77 \%)$ \\
Province & $195(19 \%)$ & $815(81 \%)$ \\
\hline Centre-Ville & $537(23 \%)$ & $1821(77 \%)$ \\
Périphérie & $309(22 \%)$ & $1065(78 \%)$ \\
Campagne & $54(21 \%)$ & $206(79 \%)$ \\
\hline Caractéristiques des individus & & $1479(76 \%)$ \\
\hline Moins de 40 ans & $474(24 \%)$ & $1157(78 \%)$ \\
Entre 40 et 60 ans & $329(22 \%)$ & $456(82 \%)$ \\
Plus de 60 ans & $97(18 \%)$ & $656(82 \%)$ \\
\hline Couple avec enfant & $147(18 \%)$ & $1378(76 \%)$ \\
Couple sans enfant & $442(24 \%)$ & $1058(77 \%)$ \\
Célibataire & $311(23 \%)$ & $569(80 \%)$ \\
\hline Moins de 3K & $140(20 \%)$ & $999(77 \%)$ \\
Entre 3K et 6K & $304(23 \%)$ & $1524(77 \%)$ \\
Plus de 6K & $456(23 \%)$ & \\
\hline
\end{tabular}

Tableau 2 : Statistique descriptives (variables hédoniques et sociodémographiques)

Seuls les bénéfices perçus des agents immobiliers affichent des différences significatives entre les deux groupes, comme le montre le tableau 3.

\begin{tabular}{lccc}
\multicolumn{1}{c}{ Bénéfices perçus } & C2C & AI & t value \\
\hline Matching & $3,3(1,7)$ & $4,5(1,7)$ & $19,0 * * *$ \\
Réassurance & $3,3(1,7)$ & $4,7(1,7)$ & $21,9 * * *$ \\
Gain de temps & $2,9(1,7)$ & $4,5(1,7)$ & $23,7 * * *$ \\
Contraintes mini. & $3,4(1,8)$ & $4,5(1,7)$ & $17,2 * * *$
\end{tabular}




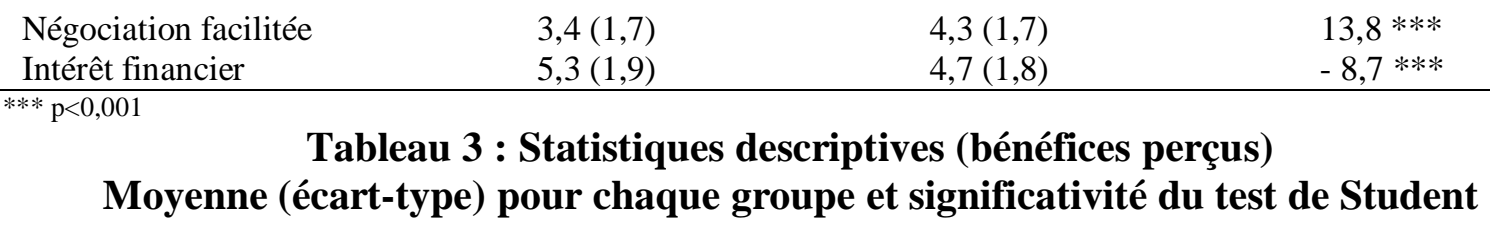

Afin d'explorer plus précisément l'effet de ces variables sur le choix d'un mode de transaction particulier, un modèle Logit est mis en î uvre. La variable dépendante expliquée concerne la probabilité d'avoir recours à un agent immobilier (groupe AI) plutôt qu'à une solution directe sans intermédiaire (groupe $\mathrm{C} 2 \mathrm{C}$ ), en fonction des variables explicatives identifiées. Le modèle de régression logistique suivant est donc établi :

$$
\mathbb{P} A I=1 \mid X=x)=\frac{\exp (\beta X+\beta)}{1+\exp (\beta X+\beta)}
$$

Où :

ÅAI est une variable aléatoire binaire qui représente le fait d'avoir (ou non) signé une transaction immobilière avec intermédiaire :

- $\mathrm{AI}=1$ : représente le fait dôvoir effectué une transaction avec un intermédiaire

- $\mathrm{AI}=0$ : représente le fait dôvoir effectué une transaction sans intermédiaire

AX est un vecteur de variables aléatoires (de réalisation $\mathrm{x}$ ), représentant l'ensemble des variables indépendantes observées.

Åb est le vecteur des coefficients estimés associés aux variables $\mathrm{X}$.

- $\quad \beta$ est la constante de régression

\section{-4 - \\ Résultats}

La première série de résultats ( $c f$. Tableau 4 Modèle 1$)$ correspond à lônalyse du recours aux agents immobiliers ( $v s$. aux particuliers) en fonction dône part des caractéristiques physiques et spatiales des logements, et dôutre part des caractéristiques sociodémographiques des individus impliqués dans la transaction. 


\begin{tabular}{|c|c|c|c|c|c|c|}
\hline \multirow{2}{*}{ Variable } & \multicolumn{3}{|c|}{ Modèle 1} & \multicolumn{3}{|c|}{ Modèle 2} \\
\hline & Coefficient & $Z$ value & $\operatorname{Pr}(>|\mathrm{z}|)$ & Coefficient & $Z$ value & $\operatorname{Pr}(>|z|)$ \\
\hline (Constante) & 1,77 & 8,15 & 0,00 & $-0,79$ & $-2,56$ & 0,01 \\
\hline Achat ou vente & $-0,32$ & $-3,89$ & $\mathbf{0 , 0 0}$ & $-0,58$ & $-6,11$ & $\mathbf{0 , 0 0}$ \\
\hline Maison vs. Appartement & $-0,09$ & $-0,75$ & 0,46 & $-0,12$ & $-0,90$ & 0,37 \\
\hline $\begin{array}{c}\text { Studio/ } 2 \text { pièces } \\
3 \text { pièces }\end{array}$ & $-0,08$ & $-0,78$ & 0,44 & $-0,07$ & $-0,59$ & 0,55 \\
\hline Plus de 4 pièces & $-0,06$ & $-0,58$ & 0,56 & 0,00 & $-0,02$ & 0,98 \\
\hline Paris & $-0,09$ & $-0,69$ & 0,49 & $-0,05$ & $-0,35$ & 0,73 \\
\hline Petite couronne & $-0,09$ & $-0,71$ & 0,48 & $-0,10$ & $-0,74$ & 0,46 \\
\hline $\begin{array}{c}\text { Grande couronne } \\
\text { Province }\end{array}$ & 0,19 & 1,51 & 0,13 & 0,29 & 2,07 & 0,04 \\
\hline $\begin{array}{l}\text { Centre-Ville } \\
\text { Périphérie }\end{array}$ & 0,05 & 0,56 & 0,58 & $-0,01$ & $-0,09$ & 0,93 \\
\hline Campagne & $-0,07$ & $-0,38$ & 0,71 & $-0,03$ & $-0,16$ & 0,87 \\
\hline Couple avec enfants & $\mathbf{0 , 3 3}$ & 2,64 & $\mathbf{0 , 0 1}$ & $\mathbf{0 , 2 7}$ & 1,91 & 0,06 \\
\hline $\begin{array}{c}\text { Couple sans enfants } \\
\text { Célibataires }\end{array}$ & 0,01 & 0,06 & 0,96 & $-0,06$ & $-0,59$ & 0,56 \\
\hline $\begin{array}{l}\text { Moins de } 40 \text { ans } \\
\text { Entre } 40 \text { et } 60 \text { ans }\end{array}$ & $-0,17$ & $-1,88$ & 0,06 & $-0,02$ & $-0,25$ & 0,80 \\
\hline Plus de 60 ans & $\mathbf{0 , 3 4}$ & 2,49 & 0,01 & $\mathbf{0 , 4 3}$ & 2,87 & $\mathbf{0 , 0 0}$ \\
\hline $\begin{array}{c}\text { Moins de } 3000 u ́ \\
\text { Entre } 3000 \text { et } 6000 \text { ú }\end{array}$ & 0,04 & 0,30 & 0,76 & 0,15 & 1,12 & 0,26 \\
\hline Plus de 6000 ú & 0,04 & 0,49 & 0,62 & 0,09 & 0,85 & 0,39 \\
\hline Bon matching & & & & 0,12 & 4,22 & $\mathbf{0 , 0 0}$ \\
\hline Réassurance & & & & 0,19 & 6,31 & 0,00 \\
\hline Gain de temps & & & & 0,26 & 8,08 & $\mathbf{0 , 0 0}$ \\
\hline Minimisation des contraintes & & & & 0,09 & 3,20 & $\mathbf{0 , 0 0}$ \\
\hline Négociation facilitée & & & & 0,17 & 6,14 & $\mathbf{0 , 0 0}$ \\
\hline Intérêt financier & & & & $-0,08$ & $-3,34$ & $\mathbf{0 , 0 0}$ \\
\hline
\end{tabular}

Tableau 5 : Régression logistique - Modèle 1 et modèle 2 avec bénéfices perçus 
Le premier résultat, relativement contre-intuitif en regard de la théorie économique du choix rationnel, concerne le fait que, toutes choses égales par ailleurs, les individus ont davantage recours aux agents immobiliers lorsqu'ils sont acheteurs que lorsqu'ils sont vendeurs, alors même ces acheteurs sont amenés à payer directement les frais d'agence. En revanche, les vendeurs, qui ne payent pas directement ces frais d'agence, ont davantage tendance à contractualiser via des transactions directes. Ce résultat peut s'expliquer en partie par la présence d'un nombre beaucoup plus important de biens via les agents immobiliers et démontre l'efficacité des stratégies de captation de l'offre.

Le deuxième résultat assez surprenant met en exergue le fait que les caractéristiques physiques et spatiales des biens ne constituent pas le niveau dônalyse pertinent : le recours aux agents immobiliers n'est pas davantage prévalent sur un type de bien particulier, une région géographique ou un niveau de densité spécifique : les maisons ne font pas davantage l'objet de transactions que les appartements $(\mathrm{z}=-0,75$; NS) via un agent immobilier. De même les transactions de petites surfaces ne sont pas davantage le fait de transactions directes : les individus n'ont pas davantage recours aux agents immobiliers pour les studios $(\mathrm{z}=-0,78$; NS) ou les 4 pièces ( $\mathrm{z}=-0,58$; NS). Plus encore, il n'existe pas "d'effet Paris" pour lequel on aurait pu penser à une plus forte présence des transactions directes lorsque la densité des logements est élevée : les individus n'ont pas davantage recours aux agents immobiliers pour Paris $(\mathrm{z}=-$ 0,69 ; NS) ou pour les centre-villes ( $\mathrm{z}=-0,71$; NS) par rapport aux autres lieux géographiques. Finalement, le type de logement, la taille et l'emplacement géographique n'ont donc pas d'incidences sur le mode de transaction choisi par les individus. Ces résultats montrent donc que les variables classiquement utilisées dans la littérature en économie immobilière ne sont pas celles qui déterminent la question du choix du le mode de gouvernance spécifique

Le troisième résultat concerne le fait que les variables socio démographiques apparaissent elles-aussi comme ayant peu d'influence. Le fait de bénéficier dồn revenu relativement plus élevé ne rend pas moins sensible aux frais dôgence que les autres individus et n'explique pas une éventuelle facilité du recours aux agents immobiliers ( $\mathrm{z}=0,49$; NS). Si le revenu nô pas dâffet sur le choix des individus, en revanche, le statut matrimonial et lộge semblent influer sur le choix dôn mode de transaction. En effet, le fait de vivre en couple avec des enfants $(\mathrm{z}=2,64 ; \mathrm{p}<0,05)$ semble pousser les individus à faire appel à un professionnel de lômmobilier lors de leurs transactions, sans doute pour des questions de temps disponible et d'organisation. De plus, les individus de plus de 60 ans tendent à davantage utiliser les services des intermédiaires que ceux de 40 à 60 ans $(\mathrm{z}=2,49 ; \mathrm{p}<0,05)$, résultat que l'on peut expliquer sans doute par un besoin de réassurance.

Le second modèle ( $c f$. modèle 2 Tableau 2) analyse le recours aux agents immobiliers en fonction d'une part des caractéristiques des biens et des individus précédemment étudiés et d'autre part de la perception des bénéfices que peuvent apporter des intermédiaires pour mener à bien la transaction par rapport à une transaction en directe.

Un résultat préliminaire concerne tout d'abord la stabilité des effets précédemment explicités. En plus des effets significatifs du type de bien, du statut matrimonial et de l'âge, le second modèle fournit une meilleure compréhension du recours aux agents immobiliers. Ainsi, lorsque la présence d'un intermédiaire est jugée rassurante $(\mathrm{z}=6,31, \mathrm{p}<0,000)$ et que celui-ci est susceptible de faire gagner du temps $(\mathrm{z}=8,08, \mathrm{p}<0,000)$, les individus ont logiquement tendance à davantage utiliser les services d'intermédiation : le recours aux agents immobiliers s'explique donc en priorité par l'idée de diminuer les "risques perçus" liés à la transaction et par l'idée d'une transaction qui va rapidement se concrétiser, ce que la littérature en économie de l'immobilier confirme (YAVAS, 1994). 
Dans une moindre mesure, mais de manière toujours significative, lorsque l'agent immobilier est perçu comme permettant de minimiser les contraintes $(z=3,20, p<0,000)$, que son intervention peut faciliter les négociations avec l'autre partie $(\mathrm{z}=6,14, \mathrm{p}<0,000)$ ou qu'il a toutes les compétences pour trouver un logement (ou un acheteur) qui corresponde à ses attentes $(\mathrm{z}=4,22, \mathrm{p}<0,000)$, alors il aura significativement davantage recours aux services d'intermédiation. A l'inverse, lorsque les services de lâgent immobilier ne sont pas jugés financièrement intéressants, les individus ont tendance à privilégier la solution directe $(\mathrm{z}=-3,34$, $\mathrm{p}<0,00)$. L'ensemble des bénéfices identifiés dans la phase exploratoire est donc bien de nature à expliquer pourquoi il existe des intermédiaires sur le marché immobilier. Les individus qui y ont recours en perçoivent réellement un certain nombre d'avantages.

L'introduction des bénéfices perçus dans le modèle explicatif est pertinent non seulement d'un point de vue théorique mais aussi d'un point de vue statistique : le modèle 2 apparait statistiquement plus performant que le modèle 1. En effet, en appliquant les modèles précédents à lânsemble des individus, la probabilité que chaque individu ait recours à un agent immobilier est estimée à partir de lấquation (1). Après avoir estimé les probabilités pour chaque individu à partir des 2 modèles, il convient de définir une règle de décision qui, à partir dôn seuil de probabilité, détermine si lôndividu présente les caractéristiques dôvoir recours ou non à un intermédiaire. La règle de décision pour le seuil de probabilité est définie sur la part de marché des intermédiaires immobiliers observée sur notre échantillon dấtude (i.e. 77\%) : Si la probabilité estimée à partir de nos modèles est inférieure à $77 \%$, alors on postule que lôndividu nôtilise pas les services dồn professionnel pour réaliser sa transaction, et inversement dans le cas d'une probabilité supérieure à ce taux empirique. La performance prédictive de ce type de modèles est évaluée à partir de la mesure du niveau dôssociations entre la probabilité prédite et la valeur réelle de la variable dépendante.

Les mesures dôassociations opérées montrent alors que le modèle 2, intégrant les bénéfices perçus, permet dôbtenir une prédiction significativement meilleure que le modèle 1 : le modèle 2 permet dôbtenir une prédiction sur le choix du mode de transaction pour $78 \%$ de lấchantillon (vs $68 \%$ pour le modèle 1 sans les bénéfices perçus). La perception des bénéfices se révèle significativement explicative du choix du mode de transaction observé sur le marché immobilier résidentiel français et améliore donc la compréhension du mode de transaction constaté sur un marché.

\section{- 5 - \\ Discussion, limites et voies de recherche}

L'objectif de cette recherche était d'étudier les déterminants du recours aux solutions intermédiées, i.e. les agents immobiliers par rapport aux solutions non intermédiées, i.e. les transactions directes, dans un contexte de généralisation d'internet et dônn éventuel renforcement de la possibilité pour chaque acteur économique de contractualiser directement. Une importante base de données a été recueillie afin de mieux comprendre le comportement des acheteurs et des vendeurs.

Premier élément de cette recherche, sur notre échantillon, la structuration du marché se révèle identique sur tout le territoire : en intégrant la part des contrats signés par les notaires, on obtient les parts de marchés de $68 \%$ pour les agences immobilières, $19 \%$ par des transactions directes via internet et $13 \%$ autres (notaires, amis, etc.). Dans une perspective de représentativité, du fait d'un biais relatif au mode de collecte par internet, l'échantillon a été redressé suivant la typologie connue du parc de logement, i.e. en suivant la distribution des biens en France par nombre de 
pièces et par la localisation (source : INSEE, recensement de la population 2008). Les résultats se révèlent similaires. Les individus n'ont pas davantage recours aux transactions directes lorsqu'ils habitent en centre-ville ou à Paris, et ils n'ont pas davantage recours aux agents immobiliers lorsqu'ils habitent à la campagne. Cette structuration des modes de transaction se révèle particulièrement stable sur les différentes sous populations étudiées : aucune différence significative n'apparait entre le groupe des vendeurs et celui des acheteurs, et plus généralement entre les différents segments opérationnalisés via les modalités étudiées dans cette recherche (type de biens, nombre de pièces, etc.). Ces résultats sont donc robustes et cohérents avec ceux précédemment identifiés (Violand et al, 2007).

Cette recherche a donc montré que la désintermédiation n'est pas un phénomène qui envahit et structure radicalement le marché immobilier comme avaient pu le prévoir certains auteurs (TUCILLO, 1997). Au contraire, l'arrivée d'internet offre aux agences immobilières de nouvelles opportunités pour transformer leur modèle économique en ne le fondant plus seulement sur la rente d'information mais sur une proposition de services plus globale, correspondant à des bénéfices attendus par certains consommateurs lorsque d'autres n'y voient pas d'intérêts. $\mathrm{Ce}$ modèle explique donc qu'un marché intermédié côtoie ainsi un marché non intermédié. Alors que l'analyse néo institutionnaliste inspirée de Williamson propose l'existence d'un mode de gouvernance optimal, fonction de spécificités objectives (spécificités des actifs, niveau d'incertitude, etc.), pour organiser les transactions dans un secteur, cette recherche explore une extension de cette perspective. Ce ne serait pas seulement les coûts de transactions en tant que données extrinsèques objectivables qui détermineraient la structure de marché mais peut être davantage la perception qu'en auraient les décideurs économiques et l'évaluation qu'ils feraient de l'intérêt des services d'intermédiation. La décision make or buy au niveau individuel contribuerait à expliquer la structure plutôt market ou hierarchy du secteur. L'hétérogénéité des décideurs contribuerait dès lors à expliquer les formes hybrides de transaction observées sur les marchés.

L'hétérogénéité des décideurs dans la perception des coûts de transactions et de l'avantage concurrentiel que les agents immobiliers offrent aux yeux des consommateurs se retrouve au sein des deux dimensions, processus de recherche et gestion de la transaction, identifiées dans la phase qualitative. Les différents risques perçus constituent dès lors des coûts psychologiques plus ou moins élevés pour les consommateurs. Certains attendent dès lors des intermédiaires immobiliers qu'ils leur permettent de les diminuer de manière significative. Ces coûts de transaction perçus sont donc à la fois un coût à réduire pour les consommateurs et une source de valeur potentielle pour les intermédiaires (ZAJAC, OLSEN, 1993).

Le niveau d'analyse individuel se révèle significatif et finalement plus pertinent que celui de la typologie des biens et leur emplacement géographique, ou celui des variables sociodémographiques caractérisant les individus (âge, nombre personnes dans le foyer, etc.) pour comprendre les types de modes de gouvernance observés sur un marché. Mais cette recherche n'est pas exempte de limites. La première concerne le fait que l'enquête s'est déroulée en ligne à partir d'un site internet de recherche d'information sur les prix immobiliers. Cette procédure a permis d'envoyer 180000 questionnaires avec un traitement facilité, une certaine rapidité de recueil et un coût financier limité. Ce type dânquête est cependant confronté à l'inconvénient de la représentativité des répondants. Seuls les individus, connectés à Internet et intéressés par l'information du site ont laissé une adresse mail. C'est sur cette base non parfaitement représentative de l'ensemble des transactions immobilières françaises qu'a été menée l'enquête. 75\% des français disposaient en 2011 dồne connexion Internet chez eux 
(enquête conditions de vie et Aspirations, 2011). Le biais existe dans la mesure où l'on observe des différences importantes dans la probabilité dô̂tre connecté : une personne âgée de plus de 70 ans a 5 fois moins de chance dô̂tre connectée quône personne âgée de 40 à 59 ans. Notre variable de contrôle de lộge atténue cependant cet élément.

Une seconde limite concerne la temporalité de cette enquête. Les individus ont été interrogés sur leurs perceptions des agents immobiliers après avoir mené la transaction. Dès lors les répondants seront tentés de rationaliser leur décision a posteriori en réduisant leur dissonance cognitive (Festinger, 1957). Ce mode de récolte pourrait donc introduire des biais dans la perception quônt les consommateurs sur les différents services immobiliers. Une voie de recherche consisterait ainsi à mener une étude longitudinale, questionner les individus ayant un projet, suivre l'avancement et l'évolution des perceptions et mettre en lien les éléments recueillis avec le mode de transaction finalement choisi.

Enfin, cette recherche fournit des éléments d'explication sur l'apparition d'acteurs "hybrides" : ni plateformes $\mathrm{C} 2 \mathrm{C}$, ni agences traditionnelles. Les premières à apparaître sont celles bénéficiant de l'effet pionnier et d'externalités de réseaux positives : des portails d'annonces se sont ainsi créés, assurant aux consommateurs des volumes conséquents de biens rendant plus aisé un bon matching. De mêmes les plateformes cybermédiaires, intermédiaires positionnés sur internet sans agences physiques, tendent aujourd'hui à se positionner en fonction des différents bénéfices attendus par les consommateurs. La nouvelle philosophie est que le consommateur devrait avoir la possibilité de choisir certains bénéfices et de renoncer à d'autres. Les frais d'agence sont alors directement fonction du niveau de service rendu : ils ne sont plus ex post, payés si la transaction se réalise, mais de plus en plus ex ante en fonction de ce qui est mis à disposition. Suivant cette logique de prestations, de nouvelles formules apparaissent, via des services « à la carte », facilités par l'absence de coûts fixes (agence, salariés, etc.). Se positionnant sur un axe qualité du service, ces acteurs proposent différentes formes de prestations, de lấstimation des prix des biens en ligne jusquầ lốaccompagnement juridique et administratif. Ce sont soit des sites d'intermédiation entre l'agence et le consommateur (type meilleursagents ou efficity), soit des sites $\mathrm{C} 2 \mathrm{C}$ qui mettent de plus en plus à disposition des prestations et/ou documents (payants) afin de faciliter la transaction et de rassurer le consommateur : conseils, guides juridiques, contrats, etc. Ce faisant, ils investissent le "marché de service" des agences traditionnelles. A titre d'exemple, les nouveaux acteurs peuvent proposer de ne pas "minimiser les contraintes", i.e. l'ensemble des services excepté les visites, que le consommateur effectue lui-même pour une commission réduite (aux alentours de $2 \%$ ). Le marché de l'intermédiation voit ainsi apparaître une nouvelle organisation via de nouvelles structures d'acteurs se positionnant sur un continuum entre market et hierarchy, i.e. entre les simples plateformes $\mathrm{C} 2 \mathrm{C}$ et les agences traditionnelles. La capacité de ces agences traditionnelles à occuper ces nouveaux marchés de l'intermédiation constitue sans doute pour elles un gage de survie. 


\section{Annexes 1 : Matrice des corrélations}

\begin{tabular}{lcccccc} 
Variables & $\mathbf{1}$ & $\mathbf{2}$ & $\mathbf{3}$ & $\mathbf{4}$ & $\mathbf{5}$ & $\mathbf{6}$ \\
\hline Matching (1) & 1,00 & & & & & \\
Réassurance (2) & 0,48 & 1,00 & & & & \\
Gain de temps (3) & 0,54 & 0,58 & 1,00 & & & \\
Contraintes mini. (4) & 0,46 & 0,47 & 0,55 & 1,00 & & \\
Négociation facilitée (5) & 0,25 & 0,25 & 0,29 & 0,27 & 1,00 & \\
Intérêt financier (6) & $-0,14$ & $-0,13$ & $-0,17$ & $-0,11$ & $-0,25$ & 1,00
\end{tabular}




\section{Références}

ACCARdo J, BugEJa F (2009) Le poids des dépenses de logement depuis 20 ans. INSEE.

BAEN JS, GutTERY (1997) The Coming Downsizing of Real Estate: The Implication of Technology. Journal of Real Estate Portfolio Managements 3: 1-18.

BAILEY J (1996) The Emergence of Electronic Market Intermediaries. Proceedings of the $17^{\text {th }}$ International Conference on Information Systems, Cleveland 391-399.

BAJTELSMIT VL, WorzOLA E (1997) Adversarial Brokerage in Residential Real EstateTransactions: The Impact of Separate Buyer Representation. Journal of Real Estate Research 14: 65-76.

Baligh H, Richartz L (1967) Vertical Market Structures. Boston / Mass: Allyn and Bacon.

BARYla E, Zumpano L (1995) Buyer Search Duration in the Residential Real Estate Market The Role of the Real Estate Agent. The journal of Real Estate Research 10: 1-14.

BARYla EA, Zumpano LV, ELDER HW (2000) An Investigation of Buyer Search in the Residential Real Estate Market Under Different Market Conditions, Journal of Real Estate Research 20: 75-91.

BonneVAl L (2011) Les agents immobiliers. Pour une sociologie des acteurs du marché du logement. Lyon, ENS Edition.

BONNEVAL L (2012) Évolutions du métier dâagent immobilier et marchés du logement in Etre logés, se loger, habiter, sous la direction de M. Bergé et L. Rougé, Habitat et Société, L'Harmattan, 17-24.

BROUSSEAU E (1989) L'approche néoinstitutionnelle des coûts de transaction. Revue francaise d'économie 4: 123-166.

COASE (1937) The nature of the firm. Economica 4 (November) 396-405.

Crozier DA, MCLEan F (1997) Consumer Decision-Making in the Purchase of Estate Agency Services. The Services Industries Journal 17: 278-293.

Elder HW, ZuMPANO LV, BARYla EA (2000) Buyer Brokers: Do they make a Difference, Their Influence on Selling Price and Search Duration. Real Estate Economics 28: 337-362.

FISHBEIN M, AJZEN I (1977) Attitude-behavior relations: A theoretical analysis and review of empirical research. Psychological Bulletin 84: 888-918.

HACKET S (1992) A comparative analysis of merchant and broker intermediation. Journal of Economic Behavior and Organization 18: 299-315. 
HÄNCHEN T, UNGERN-STERNBERG T (1985) Information Costs, Intermediation and Equilibrium Price. Economica 52: 407-419.

HeMPEl DJ, JAIN SC (1978) House Buying Behavior: An Empirical Study in Cross-Cultural Buyer Behavior. Real Estate Economics 6: 1-21.

Hiscock, R KeARns A, Macintyre S, Ellaway A (2001) Ontological security and psychosocial benefits from the home: qualitative evidence on issues of tenure. Housing, Theory and Society 18: 50-66.

Johnson JM, NourSE HO, DAY E (1988) Factors Related to the Selection of a Real Estate Agency or Agent. Journal of Real Estate Research 3: 109-118.

Jud GD, Frew J (1986) Real Estate Brokers, Housing Prices, and the Demand for Housing. Urban Studies 23: 21-31.

Jud GD (1983) Real Estate Brokers and the Market for Residential Housing. American Real Estate and Urban Economics Association Journal 1: 69-82.

Jud GD, SEAKs GT, WinkLER TD (1996) Time on the Market: The Impact of Residential Brokerage. Journal of Real Estate Research 12: 447-458.

JuD GD, WinkLER DT (1994) What Do Real Estate Brokers Do: An Examination of Excess Returns in the Housing Market. Journal of Housing Economics 3: 289-295.

KAPLAN MF, WANSHULA LT, ZANNA MP (1993) Time Pressure and Information Integration in Social Judgment. In: Svenson O, MAule AJ Time Pressure and Stress in Human Judgment and Decision Making. Springer, 265-267.

KROSNICK JA (1989) Attitude importance and attitude accessibility. Personality and Social Psychology Bulletin 15: 297-308.

LARCENEUX F, PARENT H (2010) Le marketing de l'immobilier. Dunod.

LEVITT S, Dubner S (2005) Freakeconomics. Penguin books.

McKenZie D, Betts R (1996) Essentials of Real Estate Economics. Prentice Hall.

MunNEKE HJ, YAVAS A (2001) Incentives and Performance in Real Estate Brokerage. Journal of Real Estate Finance and Economics 22: 5-21.

NASAR J (1989) Symbolic meaning of housing styles. Environment and behavior 21: 235-257.

RANGAN KV, MENEZES MAJ, MAIER EP (1992) Channel selection for new industrial products: a framework, method and application. Journal of Marketing 56: 69-82.

RUBinsteIn A, WOLINSKY A (1987) Middlemen. The Quarterly Journal of Economics 102: 581-593.

SASS T (1984) The Economics of Information Intermediaries. Dissertation, University of Washington, Seattle / Wash. 
SimON H (1991) Bounded Rationality and Organizational Learning. Organization Science 2: $125 \mathrm{I} 134$.

TuCCILlO JA (1997) Technology and the Housing Markets. Business Economics 32: 17-20.

UFC Que choisir (2011), Des agences sans toit ni loi 495: 16-23.

ViOLAND W, SimON A, KASBI S (2007) Real Estate Brokers: Do They Inflate Housing Prices The case of France. Banque \& Marchés 111: 27-41.

Walker G, Weber D (1984) A Transaction Cost Approach to Make-or-Buy Decisions. Administrative Science Quaterly 29: 373-391.

WILLIAMSON OE (1975) Markets and hierarchies: analysis and antitrust implications. New York: Free Press.

WiLliamson OE (1981) The Modern Corporation: Origins, Evolution, Attributes. Journal of Economic Literature 19: 1537-1568.

YANG S, YAVAS A (1995) Bigger is Not Better. Brokerage and Time on the Market. The journal of Real Estate Research 10: 23-33.

YAVAS A (1995) Can Brokerage have an Equilibrium Selection Role ? Journal of Urban Economics 37: 17-37.

ZAJAC E. et OLSEN C. (1993) From transaction costs to transactionnal value analysis: implications for the study of interorganizational strategies. Journal of management studies 30: 131-146.

ZuMPANO LV,. EldER HW, BARYLA EA (1996) Buying a House and the Decision to Use a Real Estate Broker. Journal of Real Estate Finance and Economics 13: 169-161.

ZUMPANO LV, JOHNSON KH, ANDERSON RL (2003) Internet use and real estate brokerage market intermediation. Journal of Housing Economics 12: 134-151.

Les auteurs remercient le site meilleursagents.com et la chaire ville et immobilier de lổniversité Paris Dauphine pour leur soutien. 